http://jmscr.igmpublication.org/home/ ISSN (e)-2347-176x ISSN (p) 2455-0450 crossref DOI: https://dx.doi.org/10.18535/jmscr/v9i2.45

\title{
Prevalence of Candida albicans /Non albicans species in clinically suspected cases of vulvo vaginitis in antenatal women and their susceptibility to commonly used antifungals
}

\author{
Authors \\ Dr Thanooja.S ${ }^{1}$, Dr Preetha. ${ }^{2}$, Dr Nirmala. ${ }^{3}$, Dr Sharada Devi.KL ${ }^{4}$ \\ ${ }^{1}$ Assistant Professor, Dept. of OBG, Govt Medical College, Kollam \\ ${ }^{2}$ Assistant Professor, Dept. of Microbiology, Govt Medical College, Thiruvananthapuram \\ ${ }^{3}$ Professor and HOD, Dept. of OBG, Govt Medical College, Thiruvananthapuram \\ ${ }^{4}$ Professor and HOD, Dept. of Microbiology, Govt Medical College, Thiruvananthapuram \\ Corresponding Author
}

Dr Preetha.R

\begin{abstract}
Introduction: Vulvovaginal candidiasis is a common problem affecting reproductive aged women. Candida Albicans species were the most common pathogen implicated in these cases. But recently there has been reports of Non Albicans Candida species emerging as new pathogens from different parts of the world and they are reported to be less sensitive to the commonly used antifungal drugs. We tried to find out the most prevalent species of Candida among our antenatal population and their drug sensitivity patterns

Objectives of the study: 1) to isolate different candida species in culture from clinically diagnosed case of vulvovaginal candidiasis in reproductive aged women

2) To speciate the isolates using phenotypic characters and anti-fungal susceptibility of the isolates in vitro

Study Design: Cross sectional prospective study

Study Setting: Department of Obstetrics and Gynaecology, and Department of Microbiology, Government medical college, Thiruvananthapuram

Study Period: 2017 1st January to $31^{\text {st }}$ December

Study Population: Antenatal women with clinical diagnosis of vulvo vaginal candidiasis attending the hospital for antenatal and intrapartum care.

Results: vaginal swabs from 400 clinically suspected cases of vulvo vaginal candidiasis were collected and examined in culture for identification of Candida species.70.5\% of women belonged to 20-29 age group and 15.8\% in 30-39 year age group. 65 cultures (16.3\%) were positive for candida.Rest of the cultures were negative for fungal growth. Out of 65 cases 30(46.2\%) were Candida ALbicans species.And 35 cases( 53.8\%) were Non albicans Candida species.(NAC) Amoung the NAC, Candida tropicalis was the most predominant strain in our study population(24.6\%),followed by C. Glabrata(16.9\%) and C.Parasilosis (12.3\%) as the other isolates. Antifungal susceptibility was tested against 3 major antifungals namely fluconazole, clotrimazole and Amphotericin-B. Studies showed that drug resistance to all the 3 antifungals were present among $10 \%$ of Candida Albicans strains only. Among the NAC, all the strains were sensitive to the standard antifungals and drug resistance could not be demonstrated.

Conclusion: Among the antenatal women with vulvovaginal candidiasis, Non albicans Candida was a predominant strain and the strains were sensitiveto standard antifungals used.

Keywords: Vulvovaginal candidiasis, Candida Albicans, Non Albicans candida, Drug sensitivity.
\end{abstract}




\section{Introduction}

Approximately $75 \%$ of women experience at least one episode of vulvovaginal candidiasis and during their lifetime and nearly half of them suffer multiple episodes. ${ }^{(1)}$ About $5 \%$ of women suffer recurrent episodes of vulvovaginal candidiasis defined by more than or equal to 4 episodes in a year.(2) The majority of them are caused by Candida Albicans, however resorts of vulvovaginal candidiasis due to non-Albicans species are increasing. ${ }^{(1,3)}$

Clinical manifestations caused by Non-Albicans species is indistinguishable from candida species but they differ in their susceptibility to commonly used antifungal agents and demonstrate a drug resistance to commonly used antifungals. ${ }^{(4)}$

Virulence and antifungal susceptibility of Candida isolates differ according to the species ${ }^{(5)}$ The available therapeutic options for vulvovaginal candidiasis include imidazole and triazole agents in topical and oral forms ${ }^{(6)}$. Azoles are the treatment of choice for vulvo vaginitis in many countries, however development of resistance to these drugs have been reported from yeast isolates from vulvo vaginal candidiasis ${ }^{(7)}$.

The present study was done aiming to assess the prevalence and anti-fungal susceptibility patterns of various types of candida species in pregnant women attending hospitals for antenatal or intranatal care in our hospital.

\section{Materials and Methods}

The study was conducted jointly in the department of Obstetrics and Gynaecology and Dept of microbiology of a tertiary care teaching Institute. The protocol of the study was discussed in the Institutional Review Board. Primary objective of the study was to isolate the different candida species in culture from clinically diagnosed cases of vulvo vaginal candidiasis in reproductive age group women. Secondary objectives of the study was to speciate the isolates using phenotypic characters and to analyse the antifungal susceptibility in vitro. This was done as a cross sectional prospective study. Antenatal patients attending the antenatal OPD and in patients with clinical features of vulvo vaginal candidiasis were the subjects of the study. Consent was taken from participants after explaining about the study and procedure of sample collection.

Two vaginal were collected from posterior vaginal fornix using Sim's speculum under aseptic precautions and transported to lab in separately labelled containers. Swabs were gram stained and cultured on Sabaraud's dextrose Agar. Colonies suggestive of candida were identified and further speciated using germ tube test, chlamydospore formation, sugar fermentation and sugar assimilation test. Antifungal susceptibility in vitro was done by Kirby Bauer method using Muller Hinton agar with $2 \%$ glucose and Crystal violet for commonly used antifungals like fluconazole, Clotrimazole and Amphotericin Data was analysed using SPSS software.

\section{Results}

This study was done during 2017January to December.400 Samples from cases with clinical symptomsof vulvovaginitis were examined for identification of candida species. $70.5 \%$ of women belong to 20-29 year age group followed by 30-39 year age group $(15.8 \%)$.

The age group of the cohort (table 1)

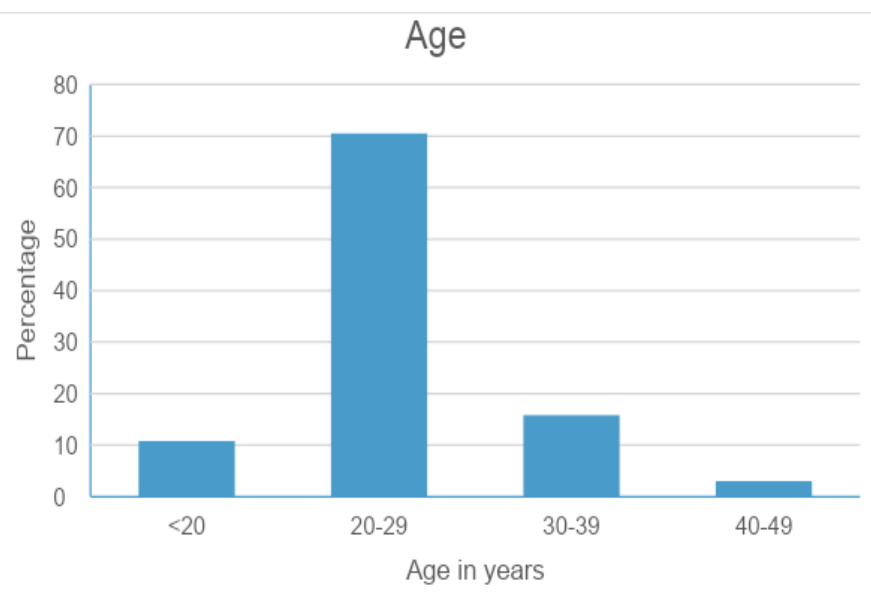

65 cultures $(16.3 \%)$ were positive for candida species. Rest were negative for fungal growth. Out of the 65 cases of candida isolated from the clinical specemens,30 (46.2\%) were Candida albicans,and 35 (53.8\%) were Non Albicans 
Candida (NAC). Among the NAC, Candida tropicalis $(24.6 \%)$, followed by Candida Glabrata
(16.9\%) and Candida Parasilosis (12.3\%) were the major isolates

Figure showing percentage of fugal culture positive isolates

\section{Culture}

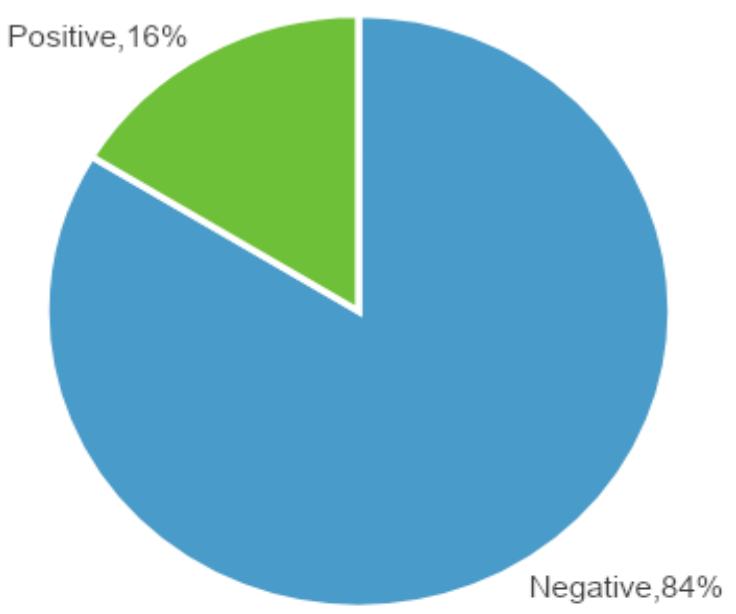

Different species isolated in culture (figure 2)

\section{Species}

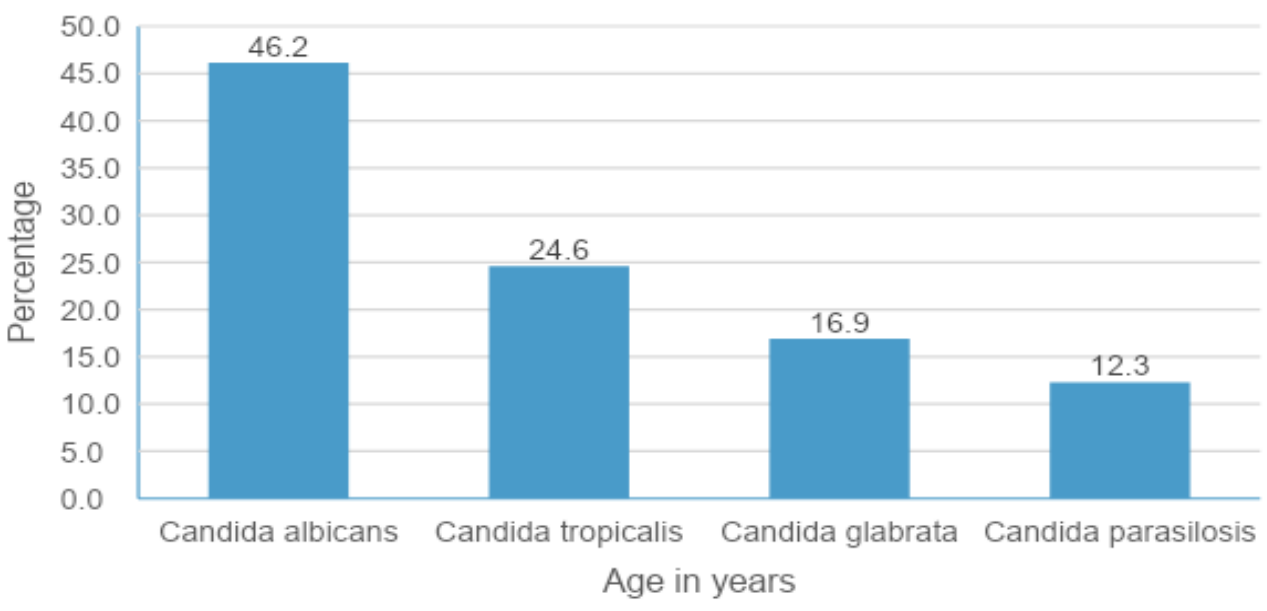

Antifungal susceptibility profile of the isolated strains were examined which shows that drug resistance was present in $10 \%$ of candida albicans species to all the three antifungals tested namely
Fluconazole, Clotrimazole and Amphotericin B.Amoung NAC species, drug resistance studies showed that All the NAC species were sensitive to the commonly used antifungals.

\begin{tabular}{|l|l|c|c|c|c|c|c|c|c|}
\hline \multirow{3}{*}{ Drug } & \multicolumn{9}{|c|}{ Species } \\
\cline { 3 - 11 } & & \multicolumn{2}{|c|}{$\begin{array}{c}\text { Candida } \\
\text { albicans }\end{array}$} & \multicolumn{2}{c|}{$\begin{array}{c}\text { Candida } \\
\text { Tropicalis }\end{array}$} & \multicolumn{2}{c|}{$\begin{array}{c}\text { Candida } \\
\text { Glabrata }\end{array}$} & \multicolumn{2}{c|}{$\begin{array}{c}\text { Candida } \\
\text { Parasilosis }\end{array}$} \\
\cline { 3 - 11 } & $\mathrm{N}$ & $\%$ & $\mathrm{n}$ & $\%$ & $\mathrm{~N}$ & $\%$ & $\mathrm{~N}$ & $\%$ \\
\hline \multirow{2}{*}{ Fluconazole } & Resistant & 3 & 10 & 0 & 0 & 0 & 0 & 0 & 0 \\
\cline { 2 - 11 } & Sensitive & 27 & 90 & 16 & 100 & 11 & 100 & 8 & 100 \\
\hline \multirow{2}{*}{ Clotrimazole } & Resistant & 3 & 10 & 0 & 0 & 0 & 0 & 0 & 0 \\
\cline { 2 - 11 } & Sensitive & 27 & 90 & 16 & 100 & 11 & 100 & 8 & 100 \\
\hline \multirow{2}{*}{ Amphotericin B } & Resistant & 3 & 10 & 0 & 0 & 0 & 0 & 0 & 0 \\
\cline { 2 - 10 } & Sensitive & 27 & 90 & 16 & 100 & 11 & 100 & 8 & 100 \\
\hline
\end{tabular}




\section{Discussion}

Vulvo vaginal candidiasis is a very common condition affecting up to $70-75 \%$ of reproductive age group women at least once in their life time. And up to $10 \%$ of these women develop recurrent VVC defined as at least 4 episodes of VVC during a 12 month period. Risk factors for VVC include extremes of age, prolonged recent broad spectrum antibiotic use, pregnancy, uncontrolled diabetes and Oral contraceptive use. Uncontrolled diabetes, with increased glycogen content, lowers the vaginal $\mathrm{pH}$ results in candida colonisation and alter the vaginal microbiotome (Vaginal dysbiosis) and predispose to infections ${ }^{(8)}$. And NAC species is found to be a significant pathogen in diabetic women with C.Glabrata and C.Tropicalis as frequent isolates. ${ }^{(9)}$

Albicans is the most common pathogenic agent of VVC, but recent studies suggest that the prevalence of NAC is increasing globally.NAC is more commonly associated with complicated $\mathrm{VVC}^{(10)}$. Among NAC, C.Glabrata, C.Krusei, C.Tropicalis and C.dubliniensis were the most frequently isolated strains. ${ }^{(11)}$. Majority of studies from different parts of the word report Candida Albicans still most frequent pathogen isolated and the prevalence ranges from $40-77 \%^{(12-14)}$. But epidemiological studies show that there is a gradual shift in the prevalence of NAC species is about $10 \%$ from 1970 s and up to $20 \%$ in 1980s.And recent study in 2018 from Nepal shows the prevalence of NAC of $33 \% .^{(15)}$

There are reports of increased resistance to commonly used anti-fungal agents like fluconazole with recurrence of VVC frequently. Most literatures are reporting resistance of NAC species to commonly used antifungals. In recent years many clinicians and microbiologists from different parts of the world have looked into and tried to study in detail regarding different species of candida as the causative of vulvo vaginal candidiasis in women. Sachin C Deodukar in their study from Maharashtra, India have reported that $36.7 \% \%$ of the isolates were candida albicans and the rest $63.3 \%$ were NAC strains ${ }^{(16)}$ In their study of NAC ,Candida tropicalis (35.1\%)was the most frequent followed by C.Glabrata $(28.1 \%)$ and C.Krusei(16.3\%)And the strains of Candida albicans showed resistance to ketoconazole in $41.7 \%$, and $33-40 \%$ cases showed resistance to other Azoles (Fluconazole and Itraconazole)..But examining cases of NAC drug resistance to standard antifungals were higher among them with $64.5 \%$ of candida glabrata and $42 \%$ of C.tropicalis being resistant to the most commonly used Azoleketoconazole. Similar reports are also found by works of Richter, Nyirjesy, and Ray $\mathrm{D}^{(7,17,18)}$

Maitrayee Sen et all reported from Chennai reported a higher prevalence of NAC species compared to Candida Albicans strain (63\% vs 37\%)(19). Drug sensitivity to standard anti fungals showed resistance to Clotrimazole in $1 \%$,miconazole $4 \%$, and $10 \%$ showing resistance to ketoconazole. This data showed that though there is a shift in the species of candida to NonAlbicans Candida species, drug resistance is not a major problem in Candida Albicans as well as NAC species isolated from Chennai.

In the present studydone among 400 cases of vulvovaginitis, 65 cases (16\%) were positive for Candida species. Among the positive cultures, $46.2 \%$ were C Albicans followed NAC species in $53.8 \%$ cases (C.Tropicalis (24.6\%), C .Glabrata (16.9\%) and C.Parasilosis (12.3\%)).And our data showed that drug resistance to antifungals was present in $10 \%$ of candida Albicans species only. Significant drug resistance was not present among strains isolated from Thiruvananthapuram which is an information relieving to the treating physician.

\section{Conclusion}

Among the antenatal women with vulvovaginal candidiasis, Non albicans Candida was a predominant strain and the strains were sensitive to standard antifungals used. Drug resistance was not a major problem among the species isolated in our institute. 


\section{Conflicts of Interest: None}

Funding: This study was funded by State Board of Medical Research.

\section{References}

1. Ferrer J. Vaginal candidosis: epidemiological and etiological factors. Int J Gynecol Obstet. 2000; 71(S1):21-7.

2. Donders GGG, Bellen G, Mendling W. Management of recurrent vulvo-vaginal candidosis as a chronic illness. Gynecol Obstet Invest. 2010; 70(4):306-21.

3. Spinillo A, Capuzzo E, Gulminetti R, Marone P, Colonna L, Piazzi G. Prevalence of and risk factors for fungal vaginitis caused by non-albicans species. Am J Obstet Gynecol. 1997 Jan; 176(1 Pt 1):138-41.

4. Subramanya SH, Baral BP, Sharan NK, Nayak N, Metok Y, Sathian B, et al. Antifungal susceptibility and phenotypic virulence markers of Candida species isolated from Nepal. BMC Res Notes [Internet]. 2017 Nov 2 [cited 2020 Aug 9]; 10. Available from: https://www.ncbi.nlm.nih.gov/pmc/articles /PMC5669004/

5. Farooqi JQ, Jabeen $\mathrm{K}$, Saeed N, Iqbal N, Malik B, Lockhart SR, et al. Invasive candidiasis in Pakistan: clinical characteristics, species distribution and antifungal susceptibility. J Med Microbiol. 2013; 62(2):259-68.

6. Watson $\mathrm{C}$, Calabretto $\mathrm{H}$. Comprehensive review of conventional and nonconventional methods of management of recurrent vulvovaginal candidiasis. Aust N Z J Obstet Gynaecol. 2007 Aug; 47(4):262-72.

7. Das KH, Mangayarkarasi V, Sen M. Antifungal Resistant in Non-Albicans Candida Species are Emerging as a Threat to Antenatal Women with Vulvovaginal Candidiasis. Biomed Pharmacol J. 2019 Sep 25; 12(2):1369-78.
8. Gunther LSA, Martins HPR, Gimenes F, Abreu ALP de, Consolaro MEL, Svidzinski TIE. Prevalence of Candida albicans and non-albicans isolates from vaginal secretions: comparative evaluation of colonization, vaginal candidiasis and recurrent vaginal candidiasis in diabetic and non-diabetic women. Sao Paulo Med J Rev Paul Med. 2014; 132(2):116-20.

9. Ray D, Goswami R, Banerjee U, Dadhwal V, Goswami D, Mandal $P$, et al. Prevalence of Candida glabrata and its response to boric acid vaginal suppositories in comparison with oral fluconazole in patients with diabetes and vulvovaginal candidiasis. Diabetes Care. 2007 Feb; 30(2):312-7.

10. Fidel PL, Vazquez JA, Sobel JD. Candida glabrata: Review of Epidemiology, Pathogenesis, and Clinical Disease with Comparison toC. albicans. Clin Microbiol Rev. 1999 Jan 1; 12(1):80-96.

11. Babic. M, Hukić M. CANDIDA ALBICANS AND NON-ALBICANS SPECIES AS ETIOLOGICAL AGENT OF VAGINITIS IN PREGNANT AND NONPREGNANT WOMEN. Bosn J Basic Med Sci. 2010 Feb; 10(1):89-97.

12. Bitew A, Abebaw Y. Vulvovaginal candidiasis: species distribution of Candida and their antifungal susceptibility pattern. BMC Womens Health. 2018 15; 18(1):94.

13. De Vos MM, Cuenca-Estrella M, Boekhout T, Theelen B, Matthijs N, Bauters T, et al. Vulvovaginal candidiasis in a Flemish patient population. Clin Microbiol Infect. 2005 Dec 1; 11(12):1005-11.

14. Shi X-Y, Yang Y-P, Zhang Y, Li W, Wang J-D, Huang W-M, et al. Molecular identification and antifungal susceptibility of 186 Candida isolates from vulvovaginal candidiasis in southern China. $\mathrm{J}$ Med Microbiol. 2015 Apr;64(Pt 4):390-3. 
15. Subramanya SH, Baral BP, Sharan NK, Nayak N, Metok Y, Sathian B, et al. Antifungal susceptibility and phenotypic virulence markers of Candida species isolated from Nepal. BMC Res Notes [Internet]. 2017 Nov 2 [cited 2020 Aug 9];10. Available from: https://www.ncbi.nlm.nih.gov/pmc/articles /PMC5669004/

16. Deorukhkar SC, Saini S, Mathew S. Nonalbicans Candida Infection: An Emerging Threat. Interdiscip Perspect Infect Dis. 2014; 2014:1-7.

17. Richter SS, Galask RP, Messer SA, Hollis RJ, Diekema DJ, Pfaller MA. Antifungal susceptibilities of Candida species causing vulvovaginitis and epidemiology of recurrent cases. J Clin Microbiol. 2005 May; 43(5):2155-62.

18. Nyirjesy P, Seeney SM, Terry Grody MH, Jordan CA, Buckley HR. Chronic fungal vaginitis: The value of cultures. Am J Obstet Gynecol. 1995 Sep 1; 173(3, Part 1):820-3.

19. Sen M, Das KH. Prevalence and resistance pattern of candida isolated from vulvovaginal candidiasis of antenatal women. Int J Clin Obstet Gynaecol. 2019 Nov 1; 3(6):268-75. 